\title{
Automatic Fuzzy-based Hybrid Approach for Segmentation and Centerline Extraction of Main Coronary Arteries
}

\author{
Khadega Khaled \\ Department of Information \\ Technology \\ Cairo University \\ Giza, Egypt
}

\author{
Mohamed A. Wahby Shalaby \\ Department of Information \\ Technology \\ Cairo University \\ Giza, Egypt
}

\author{
Khaled Mostafa El Sayed \\ Department of Information \\ Technology \\ Cairo University \\ Giza, Egypt
}

\begin{abstract}
Coronary arteries segmentation and centerlines extraction is an important step in Coronary Artery Disease diagnosis. The main purpose of the fully automated presented approaches is helping the clinical non-invasive diagnosis process to be done in fast way with accurate result. In this paper, a hybrid scheme is proposed to segment the coronary arteries and to extract the centerlines from Computed Tomography Angiography volumes. The proposed automatic hybrid segmentation approach combines the Hough transform with a fuzzy-based region growing algorithm. First, a circular Hough transform is used to detect initially the aorta circle. Then, the well-known Fuzzy c-mean algorithm is employed to detect the seed points for the region growing algorithm resulting in 3D binary volume. Finally, the centerlines of the segmented arteries are extracted based on the segmented 3D binary volume using a skeletonization based method. Using a benchmark database provided by the Rotterdam Coronary Artery Algorithm Evaluation Framework, the proposed algorithm is tested and evaluated. A comparative study shows that the proposed hybrid scheme is able to achieve a higher accuracy, in comparison to the most related and recent published work, at reasonable computational cost.
\end{abstract}

Keywords-Automatic segmentation; coronary arteries; computed tomography angiography; centerlines extraction

\section{INTRODUCTION}

Over the past 15 years, it was reported that the ischemic heart disease and stroke are the leading causes for sudden death all over the world [1]. Coronary artery disease (CAD) is a problem of having narrow (stenosis) left and right arteries, commonly known as coronary atherosclerosis disease. These arteries are responsible for providing the cardiac muscle with oxygenated blood. Unfortunately, millions of healthy-looking human being may have CAD with no symptoms [1]. Therefore, there is a crucial need to have CAD diagnosis methodologies with a high degree of accuracy. In order to achieve this goal, a computed tomography angiography (CTA) scan is employed to capture very high quality images for the heart and its coronary arteries. CTA is a type of medical exam, in which a CTA scan is combined with an injection of a contrast media to produce pictures of blood vessels in a part of a human's body [2].
The key challenges of analysis process of coronary arteries are the narrow tubular structures and the large size of 3D captured cardiac volume [3]. The radiologist takes a large time in the diagnosis process due to these two problems. Hence, the development of an automated system for quantitative vascular shape analysis, based on coronary CTA images, is crucially needed to assist the radiologist in the diagnosis of coronary atherosclerosis. These automated coronary arteries shape analysis (ACASA) systems should be able to achieve high decision accuracy at short time for the sake of patients' life. This ACASA system has mainly two phases: 1) coronary arteries segmentation; and 2) centerline extraction phases [3].

Blood vessels segmentation from medical images is an essential phase in dealing with many medical applications, such as cardiac vessel diagnosis, i.e., stenosis of coronary arteries [4]. Automatic or semi-automatic image segmentation methods [3] are useful for the isolation and shape analysis of coronary arteries captured by CTA. Automatic segmentation is the process of extracting the object boundaries automatically by a computer. On the other hand, semi-automatic segmentation refers to the process whereby this automated segmentation phase is followed by user interaction for adjustment of the segmented object's boundaries. Despite of the increased data size of medical images, the automation of the diagnosis process is required. The latest advances in computer technology and reduced costs have made it possible to develop such systems [3]. Semi-automatic segmentation methods are extremely costly in time and effort. Automatic segmentation method, if sufficiently accurate, could give faster segmentation process.

As mentioned earlier, CTA [2] is a kind of medical CT scan injection of a contrast media to produce pictures of blood vessels. The contrast of CTA is injected through an intravenous (IV) line started in patient's arm. It is a type of X-ray that uses a computer to make cross-sectional images of body organ i.e. cardiac. However, input of CTA cardiac image can be a stack of 2D slices have only two dimensions ( $\mathrm{x}$ and $\mathrm{y}$ ) called 3D cardiac volume or be a 3D image of real cardiac object has third dimension, the depth (z) [4].This third dimension allows for rotation and visualization from multiple perspectives. It is found from the literature review that the previous proposed 
schemes could be categorized based on two main factors: 1) dimensionality of the processed dataset; and 2) the level of automation of coronary arteries segmentation process. Table 1 shows the main categories of cardiac image segmentation methods. In addition, cardiac image segmentation methods could be categorized into six main methods: 1) histogram based methods; 2) statistical model based methods; 3) region based methods; 4) graph based methods; 5) deformable model based methods; and 6) atlas based methods [4]. Some of these methods are useful in coronary arteries segmentation. It is seen from the literature review that many techniques have been proposed using the 3D cardiac CTA volume dataset [5]-[10].

TABLE. I. CARdiac Image Segmentation Methods CAtegorization

\begin{tabular}{|c|c|c|}
\hline \begin{tabular}{|l} 
Dimensionality \\
Automation level
\end{tabular} & 2D & 3D \\
\hline Automatic & 2D-Automatic methods & 3D-Automatic methods \\
\hline Semi-Automatic & $\begin{array}{l}\text { 2D-Semi-Automatic } \\
\text { methods }\end{array}$ & $\begin{array}{l}\text { 3D-Semi-Automatic } \\
\text { methods }\end{array}$ \\
\hline
\end{tabular}

In [5], a hybrid proposed approach for the automatic twodimensional segmentation of coronary arteries from cardiac CTA volumes using Bayesian driven level set models and multi-scale vessel filtering. Using automation way, segmentation of the whole coronary tree from cardiac CTA volumes and the extraction process of the centerlines are describe in [6], the first steps of the segmentation algorithm consist of the detection of the aorta and the entire heart region then candidate coronary artery components are detected in the heart region after masking of the cardiac blood pools. Using the whole tree segmentation all the centerlines of the coronary arteries are extracted using a fast-marching level set algorithm. An automatic seeding method for coronary artery segmentation and skeletonization is proposed in [7], fuzzy connectedness theory is used to separate the coronary arteries from 3D CTA images based on the connectivity of the contrast agent in the vessel lumen and this step show the strength of fuzzy concept. In [8], an automatic method for extracting center axis representations centerlines of coronary arteries in contrast enhanced CTA volume scans using medialness-based vessel tree extraction algorithm which starts a tracking process from the ostia locations until all the branches are reached. Tracking method is proposed in [9] for tracking coronary arteries in CTA volume through 2D slices from ostium to the end, this method works based on the fact that each coronary artery has continuous pixels through slices from the start to the end point. In [10], volumetric region-growing algorithm used to segment the left coronary arteries tree, a sequential 3D thinning algorithm used as skeletonization method to extract arteries centerlines. Fig. 1 show the coronary ostium, the left main artery (LM), the left anterior descending (LAD) and the left circumflex (LCX) branches in anatomy representation (A) and in CT slice image (B).

In [5]-[10] most recent $2 \mathrm{D}$ automatic segmentation methods are presented. The main advantage of these methods is minimal user interaction so less time and less human effort, however, the accuracy needs to be better. Two-dimensionality segmentation methods offers short time to segment long vascular segment imaging such as the entire aorta or lower extremity arteries from the number of 2D slices. On the other hand, 3D segmentation methods better for smaller areas requiring higher spatial resolution such as carotid bifurcation from 3D image [11].

The semi-automatic approach combines the Hessian matrix based vesselness filter with two dimensional region growing algorithm for segmentation of the coronary arteries in CTA volume proposed in [12], centerlines of the segmented arteries are extracted using an in-house fast marching based method. In [13], a novel method presented for the automated extraction of coronary artery centerlines in 3D CTA image using a neural network (CNN) classifier for removing extraneous paths in the detected centerlines after using minimal path method to detect the optimal flow path of centerline. The main disadvantage of semi-automatic approaches whether 2D or 3D segmentation methods is computational cost and time for radiologist's diagnoses process and this can be effect the patient's health. Optimized particle filtering approach for the extraction of full coronary trees from 3D cardiac CTA image proposed in [14], this approach relies on a Bayesian model combining data likelihood with radius and direction priors along the coronary arteries following a centerline-based tracking design. In [15], a fully automatic coronary artery centerline tracking algorithm for 3D cardiac volume is proposed. In this algorithm, a complex continuous wavelet transform with the Gaussian kernels is used to reduce noise effect of CTA slices. Then, a multiple hypothesis tracking approach is applied to segment $3 \mathrm{D}$ vessel structures, and the tracking procedure is completed by applying a presented branch searching approach based on region growing algorithm and a mathematical morphology operation.

Finally, it could be seen from this literature that the 2D automatic methods for coronary arteries segmentation and centerlines extraction are preferable to be used in an environment that requires less time and reasonable accuracy.

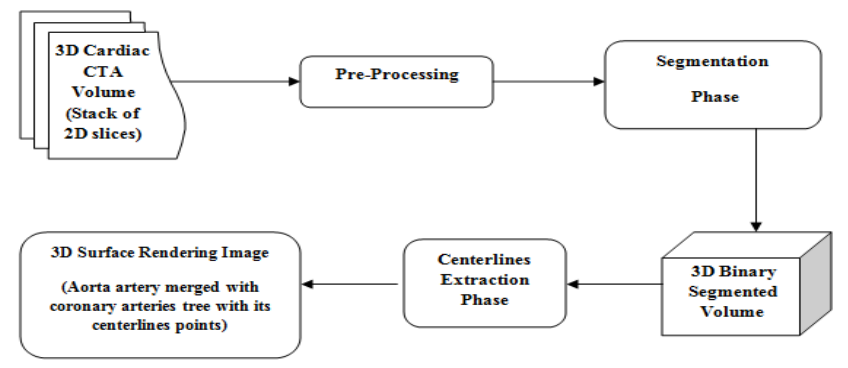

Fig. 1. Global view of hybrid proposed scheme.

It is well known that the Hough transform is an important technique used in image analysis and digital image processing as shape detection algorithm [16]. The classical Hough transform was involved for line detection in an image, but later the Hough transform has been extended to extract positions of special shapes, most ordinarily circles or ellipses. Hough transform as edge based segmentation method which can be used automatically to detect the aorta artery, that is, take a circle shape in CTA volume. The aorta is the main artery of the heart, which provides oxygenated blood to the heart and the rest of the body. Aorta artery is the root of two main coronary arteries from the original points known as "ostia points", the 
main coronary arteries are Right Coronary Artery (RCA) and Left Main artery (LM), and those two coronary arteries provide oxygen rich blood to the cardiac muscle. LM artery divides on Left Anterior Descending (LAD) and the Left Circumflex Artery (LCX) as main arteries. The main goal of the segmentation process is to partition an image into regions. As mentioned earlier, region growing is one of region-based image segmentation methods and it is found to be effective approach in blood vessels segmentation process. The idea of region growing algorithm [17] is testing the neighboring pixels of initial seed points and determines whether the pixel neighbors in $2 \mathrm{D}$ or voxel neighbors in $3 \mathrm{D}$ should be added to the region in the iteration process. The aim of applying region growing in CTA volume is extracting the coronary arterial tree in each 2D slices have coronary arteries region. In this paper, a new approach is proposed for Automatic segmentation and centerline extraction for main coronary arteries from 3D cardiac volume. In this scheme, a new 2D based region growing algorithm is proposed to be able to achieve more accurate segmented arteries at a reasonable computational cost. Impressed by the ability of fuzzy C-means clustering (FCM) technique to meet different challenges in different research fields [18], our new 2D based region growing algorithm uses the FCM for determining the seed points. This new growing algorithm is used to produce a 3D volume binary data, which leads to faster extraction process of centerlines using skeletonization approach [19] .

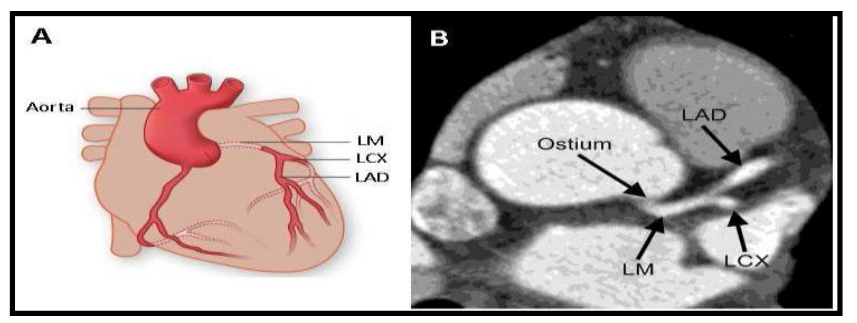

Fig. 2. (A) Aorta and LCA anatomy. (B) Example of a CT slice image showing the coronary ostium, the left main artery (LM), the left anterior descending (LAD) and the left circumflex (LCX) branches [10].

The rest of this paper is organized as: In Section 2, the overall proposed scheme is discussed. Segmentation phase is proposed in Section 3.Then centerline extraction phase is explained in Section 4. The comparisons and experimental results are presented in Section 5. Finally, conclusion and further work are in Section 6.

\section{THE PROPOSED HYBRID SCHEME}

The proposed scheme consists of two main phases, segmentation and centerlines extraction. Segmentation phase has been conducted by integrating the circular Hough transform approach with $3 \mathrm{D}$ volume region growing approach. In our region growing approach, the FCM algorithm has been utilized to detect the region growing seed points. This resulted in segmented arteries as a 3D binary volume. This binary $3 \mathrm{D}$ segmented volume simplifies the centerlines extraction phase. Therefore, in the second phase, a skeletonization method is utilized to extract the centerlines points of the 3D binary segmented volume. The main phases are depicted in Fig. 2.
The CTA scans are viewed as a series of consecutive two dimensional slices of the full 3D cardiac volume. Processing of such volumetric images on a slice by slice basis requires further processing to stack and interpolate results into 3D volume. CTA database is provided by the Rotterdam Coronary Artery Algorithm Evaluation Framework [20]. The CTA volume for each patient consist of a large number of 2D images larger than 200 slices for cardiac CTA are required to scan the complete coronary tree. In order to achieve a better computing time and memory management, first, all images are resized; the size of each axial image $512 \times 512$ is reduced to $256 \times 256$ pixels [21]. Second, pulmonary vessels are removed by a morphological erosion operator using spherical kernel with a radius of one voxel. The reason for using morphology operation is to enhance the contrast of medical images by removing noisy pixels/voxels which is essential in blood vessels segmentation to be accurate.

\section{SEgmentation Phase}

Automatic image segmentation methods are helpful for the isolation and shape analysis of coronary arteries in CTA cardiac volume. Three main automatic steps in this phase: 1) aorta detection; 2) ostia detection; and 3) region growing segmentation.

\section{A. Aorta Detection}

The segmentation of coronary arteries is introduced by an automatic detection of ascending aorta artery, which is employed as an initial mask for ostia detection. An accurate segmentation of the ascending aorta is very important for more automatic seeding of heart coronaries segmentation. These ostia points reside at the lateral sides of the ascending aorta. The main features of the ascending aorta help in detecting and distinguishing it from the other similar structures found in a CTA image slice such as descending aorta. Two main features of the ascending aorta are shape and diameter size. The ascending aorta has a circular shape [22] that expands from the aortic arch down to the aortic root. The ascending aorta diameter in healthy patients is less than $2.1 \mathrm{~cm} / \mathrm{m} 2$, while the diameter of the descending aorta is less than $1.6 \mathrm{~cm} / \mathrm{m} 2$, so the ascending aorta diameter is bigger than the descending aorta diameter [23]. Depending on this features the proposed method segmenting the ascending aorta partially using circular Hough transform then full segmented with coronary arteries segmentation process using region growing. The partially segmented part of ascending aorta circle in the first several axial slices is used to detect the ostia points to segment the whole aorta with main arteries tree. Aorta detection is performed slice by slice to segment the ascending aorta from consecutive CTA image slices until the difference of the distance of the circle centers between two successive slices is equals to zero as stop condition, (1) :

$$
\mathrm{d}=\sqrt{(C x(i)-C x(i-1))^{2}+(C y(i)-C y(i-1))^{2}}
$$

Where, $C x$ and $C y$ are vectors contain the $\mathrm{x}$ coordinates and $y$ coordinates of centers of detected i circles, $d$ is Euclidean distance. It is the distance measure between two aorta circles centers of two successive slices to select the detected aorta 
circle in target slice. Target slice used to begin segment the coronary arteries. Fig. 3(b) shows example of the preprocessing result of one original slice Fig. 3(a) and 3(c) for aorta detection result.

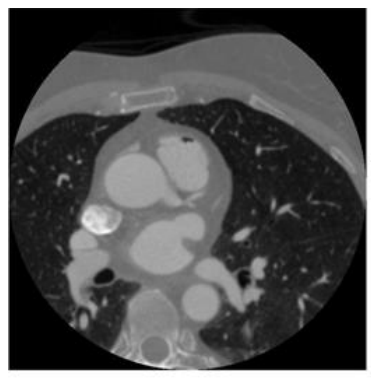

(a)

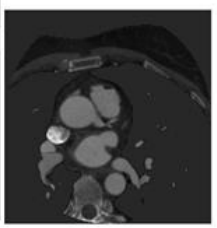

(b)

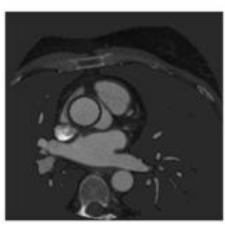

(c)
Fig. 3. (a) original slice, (b) preprocessed slice and (c) Aorta detected circle.

Once the circle of the aorta is determined, ostia points can be detected using FCM. The extracted aorta circle points from circular Hough transform help FCM technique to detect the accurate ostia points from all circle points as explained in the following.

\section{B. Ostia Detection}

After the circular region of the aorta is segmented, the ostia point of the left and right coronary trees can be detected. The segmentation of the coronary arteries is preceded by an automatic detection of the coronary ostia in which the origins of the coronary arteries is located. This method is initialized by automatically placing a single seed point in the ascending aorta on top of each coronary ostia. The main focus of this paper is that the coronary segmentation starts from the automatically detected ostia locations. More specifically, the extraction of coronary arteries centerlines relies on the vessel segmentation algorithm which starts from each ostia points and works only inside the heart mask. The ostia locations, opening points of left and right main coronary artery from the aorta are detected through an angle search regions. This regions defined in the polar coordinate system [21] is used to distinguish between the ostia of the left and right coronary trees. Based on the normal anatomy of the coronary arteries, the range of $\mathrm{OL}$ for the left Ostia $\epsilon\left[-90^{\circ}, 45^{\circ}\right]$ and for the right Ostia $\epsilon\left[45^{\circ}, 135^{\circ}\right]$. FCM [18] used here to detect optimum left ostia point and right point in $\Theta$ range from $-90^{\circ}$ to $135^{\circ}$ with two centers. It is based on minimization of the following objective function:

$$
J_{m}=\sum_{i=1}^{N} \sum_{j=1}^{C} u_{i j}^{m}\left\|X_{i-} C_{j}\right\|^{2}, 1 \leq \mathrm{m}<\infty
$$

Where, $m$ is any real number greater than $1, u_{i j}$ is the degree of membership of $x_{i}$ in the cluster $j, x_{i}$ is the $i$ th of $\mathrm{d}$ dimensional measured data, $c_{j}$ is the d-dimension center of the cluster, and $\|*\|$ is any norm expressing the similarity between any measured data and the center. Fuzzy partitioning is carried out through an iterative optimization of the (2) shown above, with the update of membership $u_{i j}$ and the cluster centers $c_{j}$. FCM implemented on the measured data extracted from the aorta detection part, where $x_{i}$ contains the $x$ and $y$ coordinates of points in $\Theta$ range of detected aorta circle.

\section{Region Growing segmentation}

Region growing method integrates only the neighboring voxels that satisfies a homogeneity criterion starting from an initial set of one or more seeds. Substantially, a region growing algorithm begins at the seed points and increases iteratively the size of the seeds check region. While the region grows, the algorithm has to decide which pixels/voxels are included into the segmented object and which are not. This decision depends on a similarity measure and the precise segmentation result is dependent on the choice of seed points. The centerline tree of the coronary arteries is computed through the region growing algorithm which has started a segmentation process from the coronary ostia locations detected using FCM as seed points until all the main vessels are extracted. Beginning from this seed points, axial aortic cross-sections are subsequently segmented by a $2 \mathrm{D}$ region growing technique. The coronary arteries are the only tubular structures that originate from the aorta. Segmentation of a thinner and a smaller object of the main coronary arteries is accomplished using a region-growing algorithm starting from the two seed points already detected using FCM on the aorta wall, a region growing is achieved for each segmented 2D cross section. The final output in such regions is this derived from the continuous vessel axis based on the cross sections. The result of region growing segmentation process consider as 3D binary volume of segmented $2 \mathrm{D}$ slices that contain the segment regions of main coronary arteries. Fig. 4 shows the 3D segmented binary volume that contains 2D slices of the main coronary arteries has all been successfully segmented: LM, LAD and LCX.
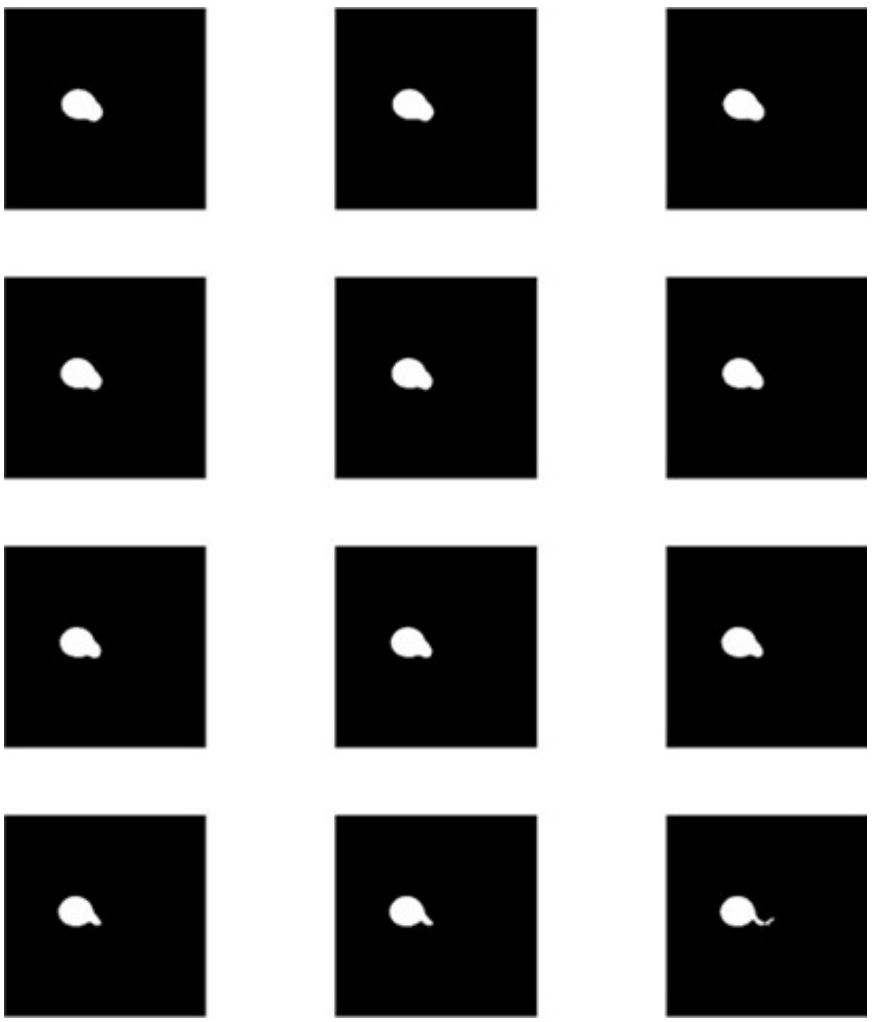

Fig. 4. 3D binary segmented volume. 


\section{CENTERlines EXTRACTION PHASE}

There are a variety of methods proposed for image skeleton extraction. The suitable technique to extract the skeleton of segmented volumetric data especially for the binary segmented volume of $3 \mathrm{D}$ tubular object is iterative thinning because it is normally only applied to binary images, and produces another binary image as output. The 3D Thinning is an iterative layer by layer erosion technique for producing medial axis i.e. skeleton from $3 \mathrm{D}$ objects. A $3 \mathrm{D}$ binary volume is a mapping process that assigns the value of 0 or 1 to each point in the $3 \mathrm{D}$ space. The input of thinning operation is 3D binary volume representing a segmented voxel level tree object to extract its centerlines by iteratively deletes or removes some object points that changes some " 1 " points to zero until it produces only one voxel wide centerlines directly. The proposed algorithm by Lee, et al. [24] used to extract the centerline of the segmented coronary arteries in 3D binary volume using parallel thinning algorithm. With the $3 \mathrm{D}$ volumetric data at hand, it is certainly useful to create a 3D view of the coronary arteries. Once segmentation is achieved using region growing, coronaries can be visualized as 3D surfaces, and various measurements can be performed conveniently in a 3D sense. The final output of proposed scheme is $3 \mathrm{D}$ view of aorta artery merged with coronary arteries tree with its centerlines points. Fig. 5 shows the 3D segmented left coronary tree centerlines extracted and visualized with the aorta. Fig. 5(a) shows the left coronary tree, and 5(b) shows the left coronary tree and right coronary artery, respectively. Finally, Table 2 contains the Pseudo code of the proposed fuzzy-based hybrid scheme.

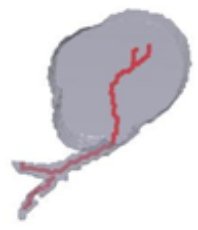

(a)

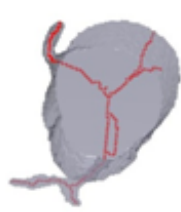

(b)
Fig. 5. (a) 3D surface rendering of LM, LAD and LCX with aorta. (b) 3D surface rendering of LM, LAD, LCX and RCA with aorta.

TABLE. II. PSEUdo CODE OF THE PROPOSED HYBRID MOdEL

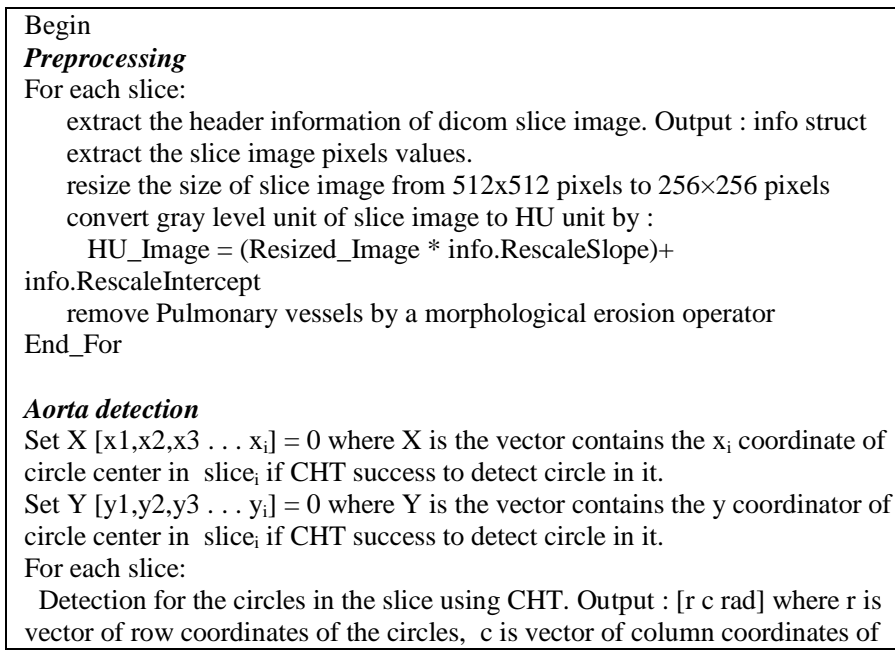

the circles and rad is vector of radiuses of the circles.

Set $\mathrm{X}(\mathrm{i})=\mathrm{c}(\mathrm{i})$

Set $Y(i)=r(i)$

Check largest diameter to differ the ascending and descending aorta.

Output : aorta circle

Check the difference of the distance of the aorta circles centers between two successive slices is equal zero as stop condition Eq. (1). Output :Initial segmentation of aorta circle

\section{Ostia Detection}

Set the left Ostia $\Theta L \in\left[-90^{\circ}, 45^{\circ}\right]$ and the right Ostia $\Theta R \in\left[45^{\circ}, 135^{\circ}\right]$ For each $\Theta=-90: 1: 135$

Get All the $x_{i}$ coordinate and the $y_{i}$ coordinate in each $\Theta$ range by : $\mathrm{x}_{\mathrm{i}}=\mathrm{X}(\mathrm{i})+\operatorname{rad}(\mathrm{i}) * \operatorname{cosd}(\Theta)$

End_For $y_{i}=Y(i)+\operatorname{rad}(i) * \operatorname{sind}(\Theta)$

Set All_Points $=\left[\mathrm{x}_{\mathrm{i}}, \mathrm{y}_{\mathrm{i}}\right]$

Clustering for the All_Points data using FCM using Eq(2). Output : the left and right ostia points.

End_For

\section{Region growing segmentation}

Apply 2D region growing algorithm which has started a segmentation process from the coronary Ostia locations detected using FCM as seed points until all the main vessels are extracted. Output: 3D binary volume contains the segmented aorta with main coronary arteries.

\section{Centerlines extraction}

Apply Lee $e t$ al. algorithm [24] to extract the centerlines points of the segmented coronary arteries in 3D binary segmented volume using parallel thinning algorithm.

End

\section{COMPARISONS AND RESULTS}

The proposed scheme was implemented using MATLAB. The described methods were applied to segment the three main coronary arteries (LAD, LCX and RCA) and extract its centerlines. The 32 CTA datasets divided into training and testing datasets. The datasets were provided by [20] as mentioned before. Datasets acquired from two 64-slice scanners were randomly selected and included in this database, 20 cases from Sensation 64, and 12 cases from Somatom Definition (Siemens Medical Solutions, Forchheim, Germany). Image quality was scored as: 1) poor, which refers to presence of image-degrading artifacts and evaluation only possible with low confidence and they are 6 cases; 2 ) moderate, which refers to presence of artifacts but evaluation possible with moderate confidence are 11 cases; and 3) good, which refers to the absence of any image degrading artifacts related to motion and noise and they are 15 cases.

The evaluation focuses on two main categories: the overlap between the automatically created centerline and the reference centerline and the average distance between those two centerlines. The error measurements proposed in [20] are overlap (OV), overlap until first error (OF), overlap with the clinically relevant part of the vessel (OT), and average inside (AI). The proposed hybrid scheme is evaluated in a similar way as done in [25]. These measurements are based on point-topoint correspondence between the detected centerline and the ground truth. A centerline point is claimed to be detected correctly if its distance to the corresponding ground truth point is not more than a threshold which is set to the radius at that point [20]. Instead of annotating the radius at each centerline point, we set the threshold to $2.5 \mathrm{~mm}$, which is approximately 
the radius of the proximal segment of a coronary artery [25]. This threshold used to calculate OV and OF measurements. The overlap for the clinically relevant part (OT) calculated using the distal segment with a radius less than $0.75 \mathrm{~mm}$ and this threshold is excluded in [20].

TABLE. III. COMPARISON OF EVALUATION RESULTS OBTAINED BY THE PROPOSED METHOD WITH AND WITHOUT FCM ALGORITHM

\begin{tabular}{|c|c|c|c|c|}
\hline Method & OV(\%) & OF(\%) & OT(\%) & $\mathbf{A I}(\mathbf{m m})$ \\
\hline $\begin{array}{l}\text { Proposed } \\
\text { scheme } \\
\text { without } \\
\text { FCM }\end{array}$ & $85 \%$ & $64 \%$ & $88 \%$ & $0.4 \mathrm{~mm}$ \\
\hline $\begin{array}{l}\text { Proposed } \\
\text { scheme with } \\
\text { FCM }\end{array}$ & $91 \%$ & $82 \%$ & $94 \%$ & $0.2 \mathrm{~mm}$ \\
\hline
\end{tabular}

In order to show the importance of using FCM in the proposed hybrid approach for ostia points' detection, the proposed scheme is implemented with and without the FCM technique. The 24 testing datasets have been used to calculate the above mentioned performance metrics. Table 3 contains the overall achieved values for the three arteries. It is clearly seen from Table 3 that the fuzzy-based hybrid proposed scheme is able to achieve results which are significantly much better than the proposed method without using FCM technique.

One of the advantages of the proposed approach is that the segmentation process is fully automated. Thus, the proposed hybrid method does not require any user interaction steps. The fuzzy-based segmentation process results in a 3D binary volume, which facilitate the extraction automatically of the centerlines points. This 3D binary output decreases the computational cost of the centerline extraction phase. The proposed hybrid segmentation method only depends on CHT algorithm and region growing approach after pre-processing steps which applies a morphological operation. In addition, the use of FCM technique made improvement in the segmentation phase and centerlines extraction phase. In segmentation, FCM detects the optimum seed points and that helped region growing algorithm to be accurate of segmented coronary arteries. The binary segmentation result of region growing has many advantages, fast to compute, easy to store and simple to process so it help the centerline extraction phase to be simple and fast. In centerlines extraction phase, FCM extracted the first centerline point as the accurate start point of centerline. It is known that the FCM gives best result for overlapped data set, unlike k-means where data point must exclusively belong to one cluster center, each data point is assigned with a membership to each cluster center. Main goal of centerline extraction step is tracking the center axis of the coronary arteries starting from the aorta surface to distal part of arteries. The centerlines points and radii of arteries considered important information to help radiologist to diagnose the place of stenosis. This shows the importance of being able to detect centerlines points more accurately.

Then to show the effectiveness of the fuzzy-based hybrid proposed scheme, a comprehensive comparative study have been conducted. In which, two comparisons are held between the fuzzy-based hybrid proposed scheme and most recent, as well as, related published work. First, in [25] a model-driven scheme was presented, in which the prior information embedded in a combined shape model with both heart chambers and coronary arteries are employed, and then the centerlines extracted using a machine learning based vesselness measurement. The results obtained for the 24 testing datasets are shown in Table 4. As it is seen from this table, the proposed fuzzy-based hybrid scheme achieves consistently higher accuracy than the model-driven method [25].

TABLE. IV. QuANTITATIVE COMPARISON OF THE PROPOSED METHOD AND MODEL-DRIVEN APPROACH

\begin{tabular}{|l|l|l|l|l|l|l|l|l|}
\hline & \multicolumn{3}{|c|}{ Model-driven Method [25] } & \multicolumn{4}{|l|}{$\begin{array}{l}\text { Proposed fuzzy-based hybrid } \\
\text { Method }\end{array}$} \\
\hline LAD & OV & OF & OT & AI & OV & OF & OT & AI \\
\hline LCX & $87 \%$ & $76 \%$ & $92 \%$ & $0.61 \mathrm{~mm}$ & $\mathbf{8 8 \%}$ & $\mathbf{7 6 \%}$ & $\mathbf{9 2 \%}$ & $\mathbf{0 . 3 m m}$ \\
\hline RCA & $71 \%$ & $65 \%$ & $89 \%$ & $0.49 \mathrm{~mm}$ & $\mathbf{9 4 \%}$ & $\mathbf{8 6 \%}$ & $\mathbf{9 4 \%}$ & $\mathbf{0 . 2 m m}$ \\
\hline $\begin{array}{l}\text { Over } \\
\text { ALL }\end{array}$ & $82 \%$ & $74 \%$ & $94 \%$ & $0.56 \mathrm{~mm}$ & $\mathbf{9 2 \%}$ & $\mathbf{8 5 \%}$ & $\mathbf{9 6 \%}$ & $\mathbf{0 . 3 m m}$ \\
\hline
\end{tabular}

Second, Table 5 contains the overall achieved performance metrics, using the training and testing datasets, of our fuzzybased hybrid scheme and scheme presented in [15]. In addition, Table 5 contains also the needed processing times for both schemes. As mentioned in [15], the main disadvantage of centerlines tracking algorithm is having difficulties to detect the ostium points of the coronary arteries due to the anomalous origins of coronary trees of some datasets like dataset 17 and 26. Our proposed scheme is able to overcome this problem by using FCM technique, which has better ability to detect the accurate ostia points from segmented aorta for each dataset. Hence, the proposed hybrid scheme is able to detect the center lines with a higher accuracy at a lower computational cost.

TABLE. V. Quantitative COMPaRison of THE PROPOSED Method AND CENTERLINES TRACKING APPROACH [15]

\begin{tabular}{|l|l|l|l|l|}
\hline Method & OV(\%) & OF(\%) & OT(\%) & $\begin{array}{l}\text { Processing } \\
\text { Time }\end{array}$ \\
\hline $\begin{array}{l}\text { Centerlines } \\
\text { tracking } \\
\text { method [15] }\end{array}$ & $60.3 \%$ & $81.6 \%$ & $58.5 \%$ & 8 min \\
\hline $\begin{array}{l}\text { Fuzzy- } \\
\text { based } \\
\begin{array}{l}\text { hybrid } \\
\text { scheme }\end{array}\end{array}$ & $\mathbf{9 5 \%}$ & $\mathbf{8 9 \%}$ & $\mathbf{9 6 \%}$ & $\mathbf{3 0 s}$ \\
\hline
\end{tabular}

It can be concluded from our comparative study that in quantitative experiments, the average extraction results of overlap measures and accuracy measure using our proposed scheme are better than results of model-driven approach [25] and centerlines tracking approach [15].In addition, the computational time is enhanced by using the 3D binary volume. The whole scheme takes about 30sincluding 20s for aorta detection, $1 \mathrm{~s}$ for arteries segmentation and $9 \mathrm{~s}$ for centerline extraction. For comparison, the proposed approach takes about $9 \mathrm{~s}$ to extract the centerlines points from a 3D binary volume which is already the fastest among all automatic centerlines extraction algorithms that participated in the MICCAI challenge [20]. 


\section{CONCLUSION AND FUTURE WORK}

In this paper we proposed a fuzzy-based hybrid approach for automatic coronary arteries segmentation and centerlines extraction process in CTA cardiac volume. The main arteries have been segmented from detected aorta artery using circular Hough transform. FCM has been used to detect the seeds points for region growing algorithm. Using FCM helped to extract the accurate start point of centerline. Centerlines have been extracted from a 3D binary volume of segmented arteries using skeleton based method. The proposed approach has been evaluated using 32 CTA datasets provided by the Rotterdam Coronary Artery algorithm evaluation framework. A comprehensive comparative study has been conducted. From which, it is found first that the fuzzy-based hybrid proposed scheme is outperform a non-fuzzy technique. Then, quantitative evaluation results showed that the proposed approach is capable of achieving much higher accuracy, at reasonable computational cost, for coronary arteries centerlines extraction in CTA volume in comparison to the other recent schemes. The average $\mathrm{OV}, \mathrm{OF}$, and $\mathrm{OT}$ measures for centerlines extraction results of three main arteries are $95 \%, 89 \%$ and $96 \%$, respectively of 32 datasets, while the average AI distance measure is $0.2 \mathrm{~mm}$ by comparison with the ground truth. Future work involves searching the branches of main arteries and measuring the diameters and cross sectional areas of segmented vessels at different locations, so that a quantitative estimation of stenosis can be provided.

[1] "World Health Organization." [Online]. Available: http://www.who.int/mediacentre/factsheets/fs310/en/. [Accessed: 10May-2017].

[2] R. Ambinder, D. Gladstone, R. Jones, Y. Kasamon, S. Shanbhag, and L. Swinnen, "John Hopkins Medicine," Sidney Kimmel Compr. cancer Cent.

[3] F. Kirbas, C. Quek, "A review of vessel extraction techniques and algorithms," Comput. Surv., vol. 36, no. 2, pp. 81-121, 2004.

[4] N. Shameena and R. Jabbar, "A Study of Preprocessing and Segmentation Techniques on Cardiac Medical Images," Int. J. Eng. Res. Technol., vol. 3, no. 4, pp. 336-341, 2014.

[5] Y. Yang, A. Tannenbaum, and D. Giddens, "Automatic segmentation of coronary arteries using Bayesian driven implicit surfaces," Proc. IEEE Int. Symp. Biomed. Imaging, pp. 189-192, 2007.

[6] P. Kitslaar, M. Frenay, E. Oost, J. Dijkstra, B. Stoel, and J. H. C. Reiber, "Connected Component and Morpholgy Based Extraction of Arterial Centerlines of the Heart (CocomoBeach)," Blood, pp. 1-8, 2008.

[7] C. Wang and O. Smedby, "An automatic seeding method for coronary artery segmentation and skeletonization in CTA," Insight J., 2008.

[8] H. Tek, M. a Gulsun, S. Laguitton, L. Grady, D. Lesage, and G. FunkaLea, "Automatic Coronary Tree Modeling," Midas J. - Proc. MICCAI Work. - Gd. Chall. Coron. Artery Track., p. <http://hdl.handle.net/10380/1426>, 2008.

[9] V. Mohan, G. Sundaramoorthi, A. Stillman, A. Tannenbaum, and others, "Vessel segmentation with automatic centerline extraction using tubular tree segmentation," Proc. MICCAI Work. Cardiovasc. Interv. Imaging Biophys. Model., 2009.

[10] S. Salles, F. P. Salvucci, and D. Craiem, "A reconstruction platform for coronary arteries, finite element mesh generation and patient specific simulations," J. Phys. Conf. Ser., vol. 332, p. 12048, 2011.

[11] Bouraoui, Bessem, et al. "3D segmentation of coronary arteries based on advanced mathematical morphology techniques." Computerized medical imaging and graphics 34.5 (2010): 377-387.

[12] İ. Öksüz, D. Ünay, and K. Kadıpaşaoğlu, "A Hybrid Method for Coronary Artery Stenoses Detection and Quantification in CTA Images," 3D Cardiovasc. Imaging, 2012.

[13] Funka-Lea, P. Sharma, S. Rapaka, and Y. Zheng, "Coronary centerline extraction via optimal flow paths and CNN path pruning," Lect. Notes Comput. Sci. (including Subser. Lect. Notes Artif. Intell. Lect. Notes Bioinformatics), vol. 9902 LNCS, pp. 317-325, 2016.

[14] D. Lesage, E. D. Angelini, G. Funka-Lea, and I. Bloch, "Adaptive particle filtering for coronary artery segmentation from 3D CT angiograms," Comput. Vis. Image Underst., vol. 151, pp. 29-46, 2016.

[15] N. Salehi and A. R. Naghsh-nilchi, "Computer Methods in Biomechanics and Biomedical Engineering: Imaging \& Visualization Automatic 3-D tubular centerline tracking of coronary arteries in coronary computed tomographic angiography," Comput. Methods Biomech. Biomed. Eng. Imaging Vis., vol. 1163, no. March 2017, pp. 1-12, 2016.

[16] B. M. Almezgagi, M. A. W. Shalaby, and H. N. Elmahd, "Improved Iris Verification System," vol. 14, no. 1, 2014.

[17] Fan, Jianping, et al. "Seeded region growing: an extensive and comparative study." Pattern recognition letters 26.8 (2005): 1139-1156.

[18] M. A. W. Shalaby . Thesis, "Fingerprint Recognition: a Histogram Analysis Based Fuzzy C-Means Multilevel," no. March, 2012.

[19] K. Palágyi and A. Kuba, "A Parallel 3D 12-Subiteration Thinning Algorithm," Graph. Model. Image Process., vol. 61, no. 4, pp. 199-221, 1999.

[20] M. Schaap et al., "Standardized evaluation methodology and reference database for evaluating coronary artery centerline extraction algorithms,” Med. Image Anal., vol. 13, no. 5, pp. 701-714, 2009.

[21] G. Yang et al., "Automatic centerline extraction of coronary arteries in coronary computed tomographic angiography," Int. J. Cardiovasc. Imaging, vol. 28, no. 4, pp. 921-933, 2012.

[22] S. C. Saur, C. Kühhnel, T. Boskamp, G. Székely, and P. Cattin, "Automatic ascending aorta detection in CTA datasets," Bild. für die Medizin 2008, pp. 323-327, 2008.

[23] R. Erbel and H. Eggebrecht, "Aortic dimensions and the risk of dissection.," Heart (British Cardiac Society), vol. 92, no. 1. pp. 137-42, 2006.

[24] T. C. Lee, R. L. Kashyap, and C. N. Chu, "Building Skeleton Models via 3-D Medial Surface Axis Thinning Algorithms," CVGIP: Graphical Models and Image Processing, vol. 56, no. 6. pp. 462-478, 1994.

[25] Y. Zheng, J. Shen, H. Tek, and G. Funka-Lea, "Model-driven centerline extraction for severely occluded major coronary arteries," Lect. Notes Comput. Sci. (including Subser. Lect. Notes Artif. Intell. Lect. Notes Bioinformatics), vol. 7588 LNCS, no. Lcx, pp. 10-18, 2012. 